\title{
TOWARDS EFFECTIVE PROJECT DOCUMENTATION, TRANSPARENCY, AND DATA-DRIVEN DECISION-MAKING THROUGH BIM-BLOCKCHAIN BASED APPLICATIONS
}

\author{
F. Raco ${ }^{1}$, M. Stefani ${ }^{2}$, M. Balzani ${ }^{1}$, L. Ferrari ${ }^{2}$ \\ 1 University of Ferrara, Department of Architecture, DIAPReM/TekneHub, Ferrara, Italy - (rcafbn, bzm) @ unife.it \\ 2 Harpaceas - (ferrari, stefani) @ harpaceas.it
}

Commission IV, WG IV/1

KEY WORDS: Digital documentation, BIM, Blockchain, Common Data Environment (CDE), Bigdata

\begin{abstract}
:
Even in the context of increasing digitisation, the construction sector continues to be characterised by redundancy, multiplication and, at the same time, a lack of transparency and disaggregation of data and information, leading to ineffective management of the time, costs and quality of the project life cycle. This paper shows the results of the development of an ICT application, TRL 4-5, based on the integration of Building Information Modeling and blockchain technologies and designed to foster digitisation processes in the supply chain, in the direction of greater transparency of information flows, knowledge-based organisations and decisionmaking processes based on unambiguous ordered data. Starting from a broader industrial research collaboration, the project involves a university spin-off, companies operating as system integrators and leaders in the customisation of BIM solutions for the Italian construction market. The project, launched as part of a network of public and private stakeholders established in 2019 and developed between September 2020 and March 2021, is part of a territorial development strategy financed by European Structural Funds. As a result, the research output is a prototype of ICT tool, which implements the Common Data Environment, CDE, making the life cycle management unambiguous, certified and clear. In this regard, the results of the project are meant to respond to the supply chain's need to encourage the digitisation and automation of processes, as well as to encourage the acquisition of unambiguous data, according to a big data approach.
\end{abstract}

\section{INTRODUCTION}

Increasingly, the use of Building Information Modeling (BIM) tools is demostrating that it is feasible to organise knowledge according to integrated digital information systems, supporting all phases of the construction life cycle.

Following the example of the digitisation process kicked off by the United Kingdom in 2009, a number of Member States have taken diversified actions to encourage the adoption of BIM tools, which are recognised as the most effective driver towards a more pervasive digital transformation of the sector [Daniotti, 2020]. In the national context, a similar result was pursued with the introduction of the new Public Procurement Code, Ministerial Decree 560/2017, which made the use of BIM tools mandatory for progressive works amount thresholds; currently, the compulsory requirement corresponds to contracts with a value of 15 million euro or more. [D.M. 560/2018]

Moreover, the described scenario has encouraged a general push in the sector towards the use of Building Information Modeling tools regardless of the mandatory thresholds and with reference, in particular, to intervention on the built heritage. Dedinitely, between November 2018 and December 2020, more than 110 BIM tenders were published by the Agenzia del Demanio for a value of more than 200 million euro. Among these ones, fiftyfive tenders were destined for: architectural, structural and plant engineering survey, diagnostics, and technical and economic feasibility plan for the assessment of the seismic vulnerability of the built heritage.

However, the sector continues to be characterised by the use of analogue information tools, as well as redundancy and duplication of data on the one hand, and information gaps on the other, conditions that lead to inefficiency in the design and construction processes, delays, poor risk management capacity [Nawari, 2019] and conflictual behaviour.

Contracts define the behaviour of the actors involved. Therefore, transparency and unambiguousness of information become essential requirements.

Recently and increasingly the number of tests of blockchain technologies applied to the construction sector, also integrated with BIM technologies, show the growing interest of the supply chain in the innovations offered by this technology in order to respond to structural inefficiencies in the value chain.

\section{RELATED WORKS}

The project for the development of a new integrated BIMblockchain application starts in the context of the strategic research and development objectives, identified as priorities for the next six years, within the framework of the Emilia-Romagna Region Smart Specialisation Strategy, with particular reference to the activity carried out by the Clust-ER BUILD for the regional technological district.

The Clust-ER BUILD public-private association, together with seven other regional Clust-ERs, acts as an observatory and advisor for the programming of the Regional Operational Plan for the implementation of the European Structural Funds for innovation and industrial research of Emilia-Romagna ecosystem, based on a project started more than ten years ago. A priority objective within the framework of the mentioned strategic planning is the development of integrated digital solutions and services that enable the increasingly widespread application of key enabling technologies: IoT, sensors, deep and machine learning, and so on. Big data for sustainability, Social Responsibility [Raed, 2019] and Security and Blockchain are the main challanges. 
Moreover, thanks to the presence of the European competence centre for BIG DATA, which was set up following the application of the project, supported by a public-private partnership of fifty-seven entities spread across the Regions of Emilia-Romagna, Lombardia, Piemonte, Sicily, Lazio, Marche and Trentino Alto Adige, to the European Community, it can now support large companies and SMEs in the region and beyond that are oriented towards the digitalization and analysis of big data.

\section{DEVELOPED METHODOLOGY}

This paper illustrates the results and products of the first phase of a technological roadmap, aimed at the digitalization of the construction sector, initiated by economic operators and public bodies belonging to the High Technology Network of EmiliaRomagna, which aims to transfer, among other skills, digital competences to a variety of production sectors. The company Harpaceas, leader in the development of BIM-based solutions for the construction market, and Raise>up, spin-off of the University of Ferrara, have developed an early integration solution between blockchain technology and BIM platform (CDE, Common Data Environment), which has reached a TRL $4 / 5$ level of validation, creating the innovative startup Innovation Chain.Therefore, the project fits into the primary objective of the Emilia-Romagna S3 Smart Specialisation Strategy, with reference to the 2021-2027 Cohesion Policy objectives and the UN 2030 Agenda. As it envisages, in the medium term, the involvement of several enabling technologies:
Internet of Things (IoT); Big Data; Cloud manufacturing (cloud computing). The main objective of the study, carried out with the consultancy of GFT Italia, is first of all the functional (Fig.1), technical and economic evaluation of integrated BIMblockchain systems aimed at:

- Certify the delivery and approval of BIM models in specific design phases;

- Certify status and responsibility transitions between professionals in processes;

- Manage contracting (collaborative contracts) and BIM-based work progress, including in public procurement contexts, through SmartContracts.

The functional analysis carried out in order to define the system requirements took into account:

- Level of digitisation of Italian companies, with particular reference to levels of cloud computing adoption [Bianchini, 2020];

- Domestic BIM-based market;

- Blockchain technology types (permissionless or public, permissioned, private).

As decentralized ledgers [Benahmed, 2020], based on consensus mechanisms, incentives, cryptography and peer-topeer systems, which associate units on the ledger (currency or digital transactions) the blockchains shift the aspect of security and trust from a local system, a database, to a global system.

Among the main innovations enabled by blockchain technology smart contracts play a central role in the construction market. In fact, smart contracts present themselves as the tool to reduce the processing time, validation and approval of the project and the

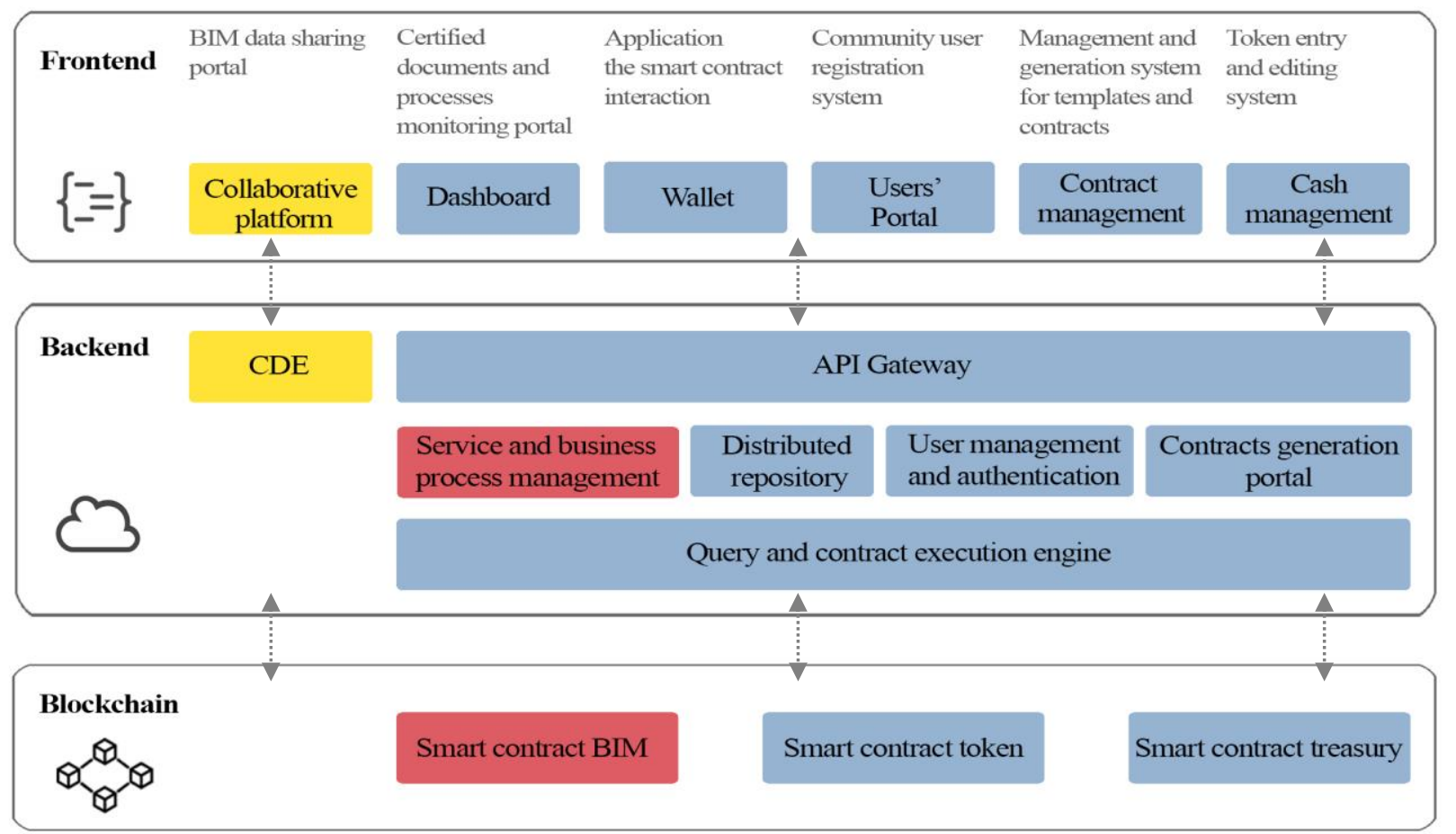

BIM solution specific components

Smart Community solution specific components

Business scenario specific components

Figure 1. The IT model components used. 
construction site, but also contractual disputes thanks to the automation of the contractual, administrative, and technical assessment phases, as well as the certification of the unicity and immutability of the information.

Accordingly, lines of research are being developed into the integration of blockchain technologies [Deloitte, 2020] and BIM tools in order to improve the coordination of project phases. This objective implies the implementation, within architectural, mechanical and plant authoring software, of plug-ins that allow the recording of annotations, as well as the validation of the geometric and informative content of both single entities of the digital model and different phases of the digital model itself [Autodesk, 2020].

However, the integration of blockchain technologies at the level of specialist development and coordination of disciplines that contribute to the definition of project requirements is subject to countless and continuous changes, unless we are in the context of a highly standardised supply chain. Unfortunately, this is not the case both in the domestic market or in the European market, which are mostly characterised by SMEs [OCSE, 2020].

The high number of transactions, corresponding to the countless modifications to which the project is subjected, corresponds to blockchain usage costs which, according to the findings of this project, could be extremely variable, as they are subject to the fluctuations of the financial market.

\subsection{Digital documentation: CDE}

The high number of design changes to which BIM models, architectural, mechanical and plant engineering models, to mention the main ones, are subject, was considered in this study as a current limitation to the widespread adoption of integrated BIM and blockchain technologies, due not only to the high costs associated, but even more to the increase in operations that this would entail in a market not yet evolved from the point of view of big data analysis.

Therefore, it was decided to operate at a higher level of project management, or project portfolio, i.e., at the level of the Common Data Environment, CDE (Fig.2), through which the different teams communicate.

The project management model (Fig.3) refers to the UNI 11337 standards and UNI EN ISO 196501-2, within which four phases are identified:

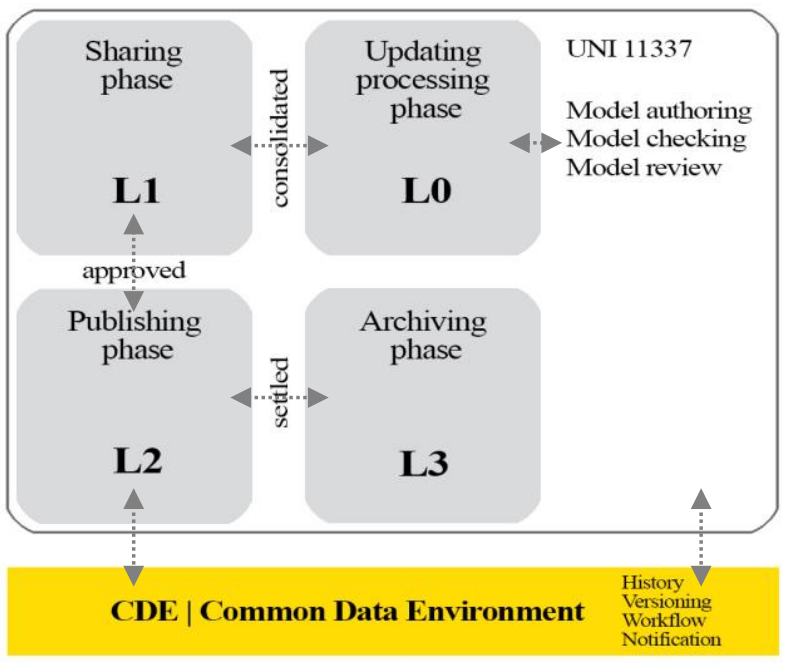

Figure 2. The Common Data Environment structure.

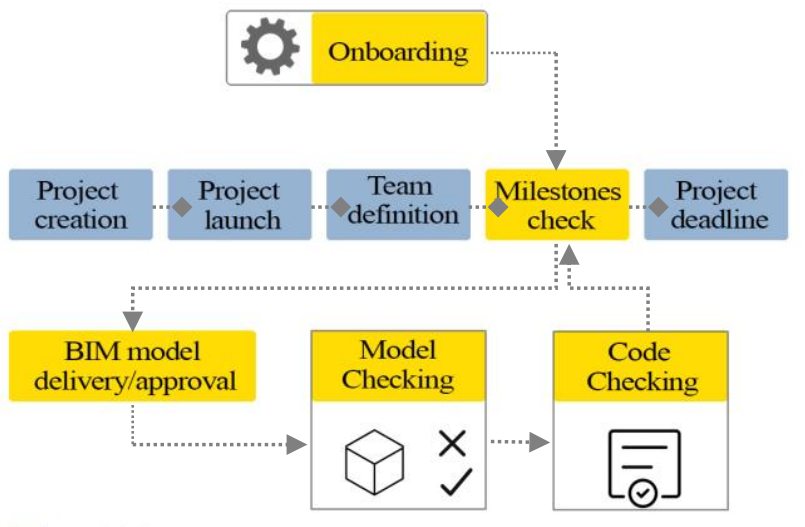

Activities

Sub-processes

Figure 3. Functional analysis "Project management": requirements.

\section{L0 - Processing/Updating Phase}

The information content is still in the "processing" phase by the specific development team, so it is not yet made available to other operators.

L1 - Sharing Phase

The information content, although considered complete for some disciplines, is not complete for all of them and therefore potentially still subject to evolution and modification.

L2 - Publication Phase

The information content is definitive and, although still susceptible to revisions, none of the actors should have any need/interest in making new changes.

L3 - Archiving Phase

Can be further distinguished: L3.V - archived but still "valid"; L3.S - archived but "outdated".

The transition from one processing status to another follows an approval process defined by the following statuses:

A0 - To be Approved

In this case the information content has not yet gone through the approval process

A1 - Approved

The information content has undergone the approval process with a positive outcome

A2 - Approved with comment

Although the information content has passed the approval process, inadequacies have been found such as to require compulsory interventions for the usability for the intended purposes

A3 - Not Approved

The approval process was unsuccessful, requiring extensive rework of the information content.

The implemented integrated system architecture takes into account, as main requirements and functionalities, the following phases

- Clash detection: a check is made for interference (conflicts) between models

- Code checking: building requirements are checked.

\subsection{Data management: reference model and functions}

Below is the model architecture where specific components of an existing BIM solution are integrated with specific elements of a blockchain-based "Smart Community" solution where the contracts (and related smart contracts) define the rules of the 


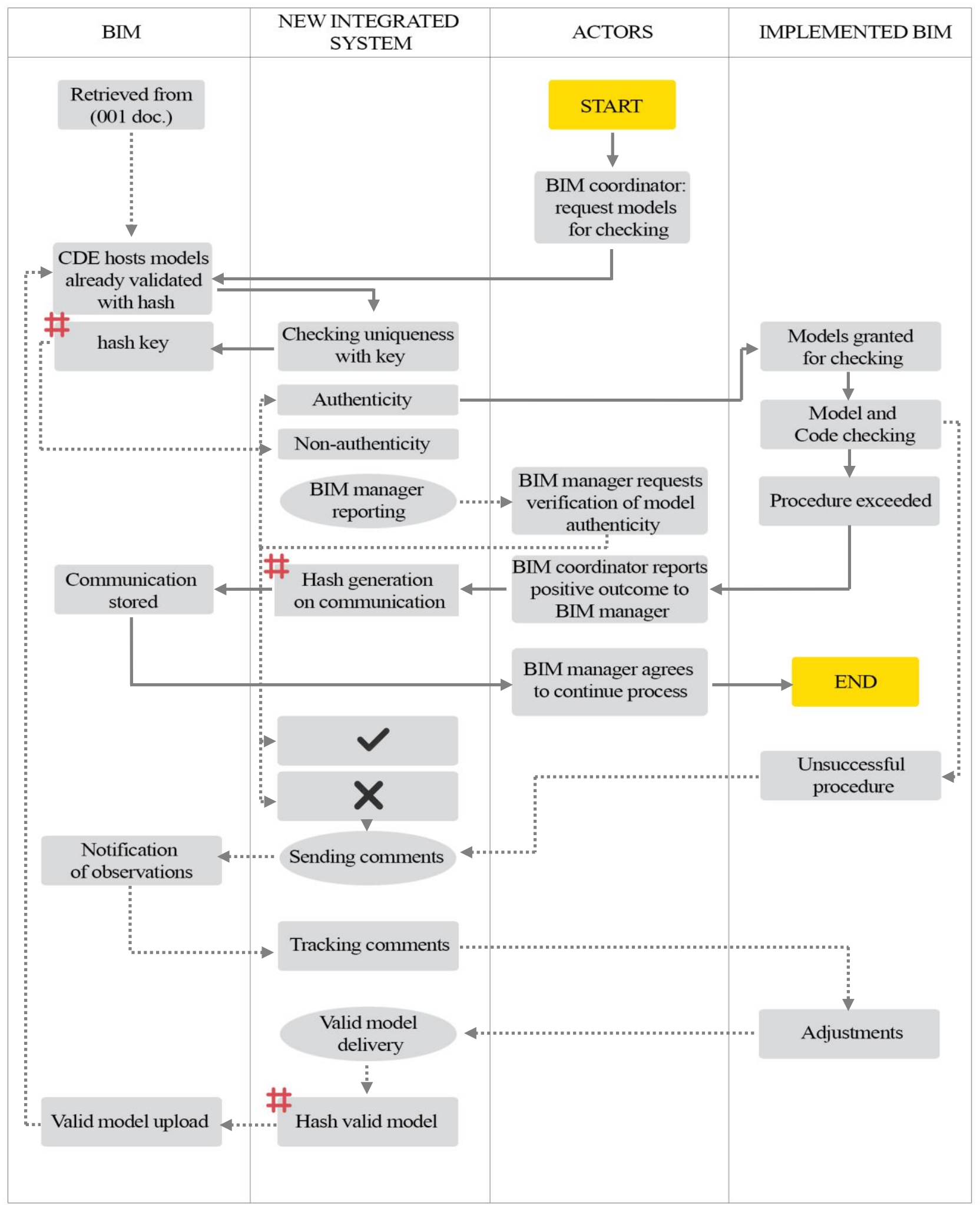

Figure 4. Verification and validation of the processing steps identified during the functional analysis. Each phase includes delivery and approval of the models, verification of interference between the models and verification of the mandatory/contractual requirements. If the HASH verification process is unsuccessful, or if the model checking and code checking process is unsuccessful, BIM resource observations are sent and saved. Definitely, BIM corrects the errors, the system receives the corrected models, converts them to HASH and loads the corrected models. Finally, the procedure is re-executed. If the procedure is successful without observations, the BIM Coordinator reports the successful outcome to the BIM Manager and a HASH key is generated on the communication and stored among the BIM resources. 
community (in this case a BIM project), the wallets uniquely identify the community participants, and the tokens represent the value generated/transferred within the community in particular steps of the BIM process. Documents and key steps in the BIM process are certified through smart contracts that generate transactions on the blockchain's distributed ledger.

These transactions are immutable, time-stamped and uniquely associated with the wallet that generated them.

The processes included in the "Project Management" perimeter are as follows:

- System Onboarding: Process consisting of the first operations to be done to access the system.

- Verification and validation of intermediate phases: Process in which the various GANTT and SAL/milestones of the project are produced, displayed and validated

- Model delivery and approval: Process in which the various project models are produced, displayed and validated

- Interference checks: Process of checking, assessing, managing and overcoming interference between models.

- Verification of mandatory requirements: The process of verifying and validating mandatory requirements.

- Verification of voluntary standards: Process of verification and validation of voluntary standards.

The platform has been designed as the integration of an innovative component of notarization on Blockchain of actions and documents within the current scenario of project management with BIM methodology. The innovative component inserts itself between the collaborative environment, through which the different project teams operate and transfer documents, and the common data repository (AcDat or CDE) and controls the "gates" of passage between the different states of processing.

The functionalities of the new component are provided both as a user interface (web/mobile) and as APIs that can be contacted by external systems. Through Smart Contracts operating on blockchain, the component stores in an unchangeable and certified way (time stamp, cryptographic signature), the main actions, the state transitions, the unique fingerprint (hash) of the documents.

The AcDat/CDE maintains its role as a common repository of data and processes, while the Collaborative Environment maintains its role as an integrated model viewer and repository navigation interface.
The developed functionalities concern (Fig.4): project creation; project start-up; definition of the project team; Onboarding; GANTT loading; GANTT updating; model delivery; model approval; model validation; validated models' extraction; insertion of the outcome of interference checking; milestone completion; smart contract project (ex. Informative Specifications).

\subsection{Frontend, backend and blockchain}

The frontend of the system includes clients specific to the BIM solution and clients specific to the Smart Community solution. Clients specific to a Smart Community solution:

- An interface for the registration of users to the Community; - A web portal for the management of smart contract templates, the generation of contracts starting from published templates and their registration on blockchain;

- An interface for the management and monitoring of treasury operations and auditing of wallet balances and transactions;

- A mobile app that provides the wallet for interaction with the blockchain and token accumulation/use;

- An interface for verification and monitoring of processes and documents certified via blockchain;

BIM solution-specific client:

Collaborative platform for the organised collection and sharing of data, information, models, objects and outputs: resulting products, component products and processes (objects, subjects, actions). Specific components of the Smart Community solution (backend) (Fig.5):

- The "Smart Contract generation engine" allows to create and manage SCs by associating the operating rules with a natural language document that represents its interface to users at the time of subscription;

- The "Smart Contract query and execution engine" allows to interact with the SCs by calling their functions to read its status or modify it, triggering the defined rules;

- The "User Management and Authentication" module provides user provisioning, user authentication and client authorisation according to the OAUTH2 standard;

- The "Service and Business Process Management" module exposes the services that implement the business logic for the Smart Community application scenario and manages the
BIM-Blockchain tool elements

CDE Web
CDE 3DViewer
CDE Identity
CDE Login
CDE API
BIM Portal
BIM Services

\section{Smart Contract Generator} Smart Contract Proxy

Project

Wallet + Wallet Services

Ethereum Client

\section{Description}

Collaborative portal and repository navigation

3D Viewer Identity Provider

Registration and login page

Repository access API

Web portal for project management developed with javascript frameworks Microservices developed with Java language

Java microservice for the creation and management of templates and Smart Contract Java microservice for Smart Contract deployment and API exposure

Smart Contract for project management and notarising process activities and documents Interface and services for signing notarisation transactions

Blockchain network access client

Figure 5. The software framework was designed following a make-or-buy analysis, whereby the system components shown in the figure were defined. 
processes by orchestrating the other modules of the platform and integrating third party systems:

- The "Api Gateway" is the module that centralises, limits and controls access to backend services by clients.

Specific components of a BIM solution:

- The "CDE" is the module for the organised collection and sharing of data related to digital models and drawings, referring to a single work or to a single complex of works.

The Blockchain provides a distributed ledger on which transactions made through Smart Contracts are recorded in a secure, certified and immutable manner.

The main Smart Contracts that define the rules and interface with the Blockchain are the following:

- "Smart Contract Token" defines the rules and records the transactions for token generation and transfer between system addresses;

- "Smart Contract Treasury" defines the rules and records the transactions for the introduction (cash-in) and redemption (cashout) of tokens in the system, with respect to current money transactions carried out on the banking circuit BIM Smart Contracts" define the rules and record the transactions related to the management of BIM-related processes.

The several new functions that have been developed include (Fig.6) "Model Delivery", "Model Approval", "Model Validation", "Extracting Validated Models", "Entering Results and Checking Interferences" to which correspond the activities of notarization of model delivery, notarization of model approval (BM and community), and notarization of results/observations checking interferences represent, in particular, the implementation with the BIM authoring environments proper. Therefore, they take into account the relationship among the representative documents of project life cycle, GANTT, Information Specifications (to mention an example), as well as type of contracts related to the project, but also the different roles and responsibilities of the project team members, in particular: BIM manager and BIM coordinator. Accordingly, the "Project" Smart Contract contains all the information to be notarised and all the operations to be carried out for a correct management of the whole Project life cycle. Notarization nodes are identified in relation to project milestones and the main stages of verification, approval and updating of the BIM model.

\begin{tabular}{ll}
\hline Information & Description \\
\hline Start date & Collaborative portal and repository navigation \\
\hline End date & \\
\hline Team & The project team members list \\
\multicolumn{1}{c}{ BIM roles } & Team member's role \\
Authoring (Arch. Emg. MEP) & Area of exprtiese of the team member \\
Wallet address & The team member's wallet address \\
\hline Current phase & Hash of current project phase \\
\hline Project status & Project status (Ongoing, Cancelled, Suspended, Closed) \\
\hline Phases/Milestones & Project phase array \\
\multicolumn{1}{l}{ Planned models and LOD } & Models planned and their LOD(Level OfDevelopment) \\
Status & Phase Status (Planned, Ongoing, Cancelled, Completed) \\
GANTT & Blockchain network access client \\
Milestone deadline & Interface and services for signing notarisation transactions \\
Check result & Outcome of the model checking \\
Delivered models & Delivered models list \\
& Model Hash \\
& Model code name \\
& Model status (To be Approved, Approved, Rejected)
\end{tabular}

Exposed methods:

Notarization of team changes

GANTT notarization

Notarization of GANTT community approval

Notarization of model delivery

Notarization of model approval (BM and community)

Notarization of results/remarks verification of interferences

Retrieval of delivered and approved models

Retrieve project information (start date, end date, team, status)

GANTT retrieval

Retrieve recorded events

Notarization of project start/closure/suspension/cancellation/reopening

Notarization of phase/milestone completion

Figure 6. Smart Contract (Project): retained Information. 


\subsection{Medium and long-term fallout}

Subsequently, engineering phases designed to reach the next levels of technological maturity of the application developed, TRL 6-9, envisage the implementation of verticalised solutions for partners and early adopters representing the main players in the construction supply chain, both in the national and international context.

The second phase of development, which is in the process of being launched, will not only allow the pursuit of data collection on the impacts on the project lifecycle management of integrated BIM-blockchain solutions, but at the same time to verify:

- opportunities and limits of integration and implementation of the different data sharing environments, $\mathrm{CDE}$, in use in the partner organisations involved;

- opportunities and limitations related to the use of the different blockchain solutions available on the market (permissionless or public, permissioned, private) and undergoing rapid evolution;

- the costs associated with the different infrastructural and technological choices identified in relation to the size of the organisations involved, the type of project portfolio managed, the types of site management involved (public, private, largescale works, interventions on existing built heritage).

Primarily, it is the mode of management of the data sharing environment, $\mathrm{CDE}$, that determines the subsequent lines of development of the integrated application.

The organisation's propensity to manage cloud-computing solutions, its willingness to adopt order management and operational models oriented to the systematic collection of data, Bigdata become essential requirements in order to implement the functionality of analytics and the planned Iot integrations [Campos, 2020].

In addition, the relationship to the next phase of the site, with respect to which the functional analysis has already been completed within this study, represents the further challenge of integrating different CDEs within a single integrated digital data collection, analysis and modelling system.

\section{CONCLUSIONS}

"Blockchain could help turn around stagnating output in construction, relative to employment, by improving contract management, enabling more transparency in supply chains, and providing the technological backbone needed to combine aspects of the Circular Economy, BIM, IoT systems and smart sensors. It adds a new layer on top of internet infrastructure for the tamper-proof exchange of value and information" [ARUP, 2017].

The possibility of integrating a higher level of automation of systems into the information infrastructure that guarantees immutability, unambiguousness and tampering of information presents itself as an opportunity for all production chains and value chains, with particular reference to the areas most characterised by controversy and corruption.

Even more, with reference to the construction sector, the technological innovation offered by the integration of blockchain technologies and enabling technologies is a tool to promote the certification of the quality of phases and products of the life cycle of the project, the work and its management. Just think of the emerging field of data collection, analysis and modelling for knowledge of the existing built heritage, increasingly characterised by the integration of integrated digital technologies.

Surveying, diagnostics, structural and seismic safety analysis, energy efficiency and risk management are just the main areas of investigation that increasingly require the adoption of protocols and standards for assessing the quality of results, the comparability of outcomes and data analysis, with a view to modelling performance based on Bigdata.

However, it is evident that some current barriers to the widespread adoption of integrated approaches to digitisation, such as those underlying the adoption of the technologies under consideration, persist.

First and foremost, unresolved disputes over regulatory issues are limiting the wider deployment of blockchain technology. At the same time, the costs associated with blockchain technology, initially conceived for the financial, banking and insurance markets, have yet to find stable and scalable conditions, in the medium term, in order to be sustained by markets characterised by lower economic-financial margins. Finally, it is necessary to consider the level of technological maturity of the individual sectors considered, with reference to specific areas and production districts. Among these, the construction sector is once again the least open to the adoption of innovative process management models and tools. Therefore, the scalability of innovation, together with the possibility of accessing dedicated resources on a competitive basis, remains, according to the authors, a central node in supporting public-private projects for the digitalisation of the supply chain.

\section{REFERENCES}

Autodesk, Integrating Ethereum with Autodesk Forge, 2020, Autodesk, pp. 1-7. Retrieved: www.constructionblockchain.org.

ARUP, Blockchain Technology. How the Inventions Behind Bitcoin are Enabling a Network of Trust for the Built Environment, 2017, Arup: London, pp. 1-72. Retrieved: www.arup.com.

Benahmed Daho, A.: "Crypto-spatial: an open standards smart contracts library for building geospatially enabled decentralized applications on the ethereum blockchain", Int. Arch. Photogramm. Remote Sens. Spatial Inf. Sci., XLIII-B4-2020, 421-426, https://doi.org/10.5194/isprs-archives-XLIII-B42020-421-2020, 2020

Bianchini, M., Know, I., Blockchain per Start-up e PMI in Italia, 2020, OECD, pp. 1-82. Retrieved: www. oecd.org.

Campos Fialho, B., Codinhoto, R., Márcio Minto, Fabricio, "BIM and IoT for the AEC Industry: A systematic literature mapping", in XXIV International Conference of the Iberoamerican Society of Digital Graphics, 2020, DOI: DOI: 10.5151/sigradi2020-54. Retrieved: https://www.proceedings.blucher.com.br/article-details/bimand-iot-for-the-aec-industry-a-systematic-literature-mapping35462.

Daniotti, B., Gianinetto, M., Della Torre, S., Digital Transformation of the Design, Construction and Management Processes of the Built Environment, 2020, Springer, pp. 1-391, ISBN 978-3-030-33570-0.

Deloitte, Deloitte's 2020 Global Blockchain Survey. From Promise to reality, 2020, Deloitte Insights. Retrieved: https://www2.deloitte.com/content/dam/insights/us/articles/660 8_2020-global-blockchain survey

/DI_CIR\%202020\%20global\%20blockchain\%20survey.pdf.

Nawari O. Nawari, Shriraam R., "Blockchain and Building Information Modeling (BIM): Review and Applications in Post- 
Disaster Recovery”, in Special Issue, BIM in Building Repair and Maintenance, 2019, MDPI, DOI 10.3390/buildings9060149. Retrieved: www.mdpi.com

Raed A. Salha, Maher A. El-Hallaq, Abdelkhalek I. Alastal, "Blockchain in Smart Cities: Exploring Possibilities in Terms of Opportunities and Challenges" in Journal of Data Analysis and Information Processing, 2019, Vol. $7 \quad \mathrm{~N}^{\circ} 3$. DOI: 10.4236/jdaip.2019.73008. Retrieved: https://www.scirp.org/journal/paperinformation.aspx?paperid=9 4347. 